\title{
Exploring Iranian $\beta$-Thalassemia major patients' perception of barriers and facilitators of adherence to treatment: A qualitative study
}

\author{
Amir Emami Zeydi ${ }^{1}$, Hossein Karimi Moonaghi ${ }^{2}$, Abbas Heydari ${ }^{3}$
}

${ }^{1}$ Ph.D. Candidate in Nursing, Department of Medical-Surgical Nursing, School of Nursing and Midwifery, Mashhad University of Medical Sciences, Mashhad, Iran

${ }^{2}$ Ph.D. of Nursing, Professor, Evidence- Based Care Research Center, Department of Medical- Surgical Nursing, School of Nursing and Midwifery, Mashhad University of Medical Sciences, Mashhad, Iran

${ }^{3}$ Ph.D. of Nursing, Professor, Evidence- Based Care Research Center, Department of Medical- Surgical Nursing, School of Nursing and Midwifery, Mashhad University of Medical Sciences, Mashhad, Iran

\section{Type of article: Original}

\begin{abstract}
Background: Despite the clinical importance of adherence to treatment in $\beta$-Thalassemia Major $(\beta-\mathrm{TM})$ patients, poor adherence remains a prevalent and persistent problem in these patients.

Objective: The aim of this study was to explore Iranian $\beta$-TM patients' perception regarding the barriers and facilitators of adherence to their treatments.

Methods: In this qualitative study, data were collected using purposeful sampling and semi-structured interviews with $18 \beta$-TM patients, 3 family members and 5 healthcare professionals (total=26) in Sari, northern Iran, between August 2015 and May 2017. Data were analyzed using conventional content analysis with the support of MAXQDA software. All the interviews were transcribed verbatim. Data were coded and grouped under specific categories.

Results: We identified and classified the perceived barriers of patients' adherence to treatment into six main categories including: (I) long distance challenges, (II) delayed occurrence of complications, (III) fatigue of healthcare providers, (IV) staff workload, (V) insufficient follow-up of healthcare workers, and (VI) busy everyday life. Also, nine main categories emerged as facilitators including: (I) concern of appearance changing, (II) outcomes of treatment, (III) good awareness, (IV) parental monitoring, (V) fear of complications, (VI) social activity, (VII) marriage as a facilitator, (VIII) friendly interaction with the medical staff, and (IX) trusting the medical staff.

Conclusion: Our findings provide a broad range of context-specific facilitators and barriers to treatment adherence perceived by Iranian $\beta$-TM patients. These factors improve our understanding of the complex and multifactorial nature of adherence to treatments. Health care providers can use these findings in designing and implementing appropriate interventions to promote $\beta$-TM patients' adherence to treatment.

Keywords: Medication adherence; Beta-Thalassemia; Patient compliance; Qualitative research
\end{abstract}

\section{Introduction}

$\beta$-Thalassemia Major ( $\beta$-TM) is the most commonly inherited hemolytic anemia. Patients with $\beta$-TM require lifelong adherence to distressing treatment regimen, including regular blood transfusions, daily chelation therapy and frequent medical supervision $(1,2)$. Like other chronic diseases requiring long-life treatment, adherence to treatment is a major concern for $\beta$-TM patients (3). It has been revealed that poor adherence to therapeutic regimen in $\beta$-TM patients is associated with poor clinical outcomes including increased risk of complications, highest risk of

\section{Corresponding author:}

Professor Dr. Hossein Karimi Moonaghi, Department of Medical- Surgical Nursing, School of Nursing and Midwifery, Mashhad University of Medical Sciences, Mashhad, Iran.

Tel: +98.5138591511, Fax: +98.5138597313, Email: karimih@mums.ac.ir

Received: August 17, 2017, Accepted: September 15, 2017, Published: December 2017

iThenticate screening: September 13, 2017, English editing: October 04, 2017, Quality control: November 12, 2017

This article has been reviewed / commented by five experts

(C) 2017 The Authors. This is an open access article under the terms of the Creative Commons Attribution-NonCommercialNoDerivs License, which permits use and distribution in any medium, provided the original work is properly cited, the use is non-commercial and no modifications or adaptations are made. 
mortality and morbidity and, consequently, increased healthcare costs. Also, poor adherence to treatment is a source of ongoing frustration for healthcare providers (4). This gives a special emphasis to maintain optimal adherence to treatment in these patients. Despite the clinical importance of adherence to treatment in $\beta$-TM patients, poor adherence remains a prevalent and persistent problem in these patients, with the reported rates ranging from 30 to 80 percent $(3,5,6)$. Although many variables have been studied to determine the predictability of patients' adherence to treatment, none of them are capable of predicting it precisely $(7,8)$. Besides, to date, cost-effective interventions to improve adherence to therapeutic regimens in $\beta$-TM patients are yet unavailable (9). Therefore, understanding the broader aspects of adherence to treatment in $\beta$-TM patients has been strongly needed (7). It has been previously demonstrated that patients' health beliefs and feelings are most common reasons for poor adherence to treatment in patients with $\beta$-TM (10). In chronic conditions, it is supposed that patients and their caregivers' belief and everyday living restrictions are of important factors associated with patients' adherence $(4,8,11)$. Therefore, it seems imperative to analyze the experiences of patients and caregivers prior to developing appropriate interventions to enhance patients' adherence to treatment, since they play a central role in the adherence process (12). In this regard, the qualitative methods can provide a deeper understanding of patients' and caregivers' experiences and perceptions. Therefore, these methods are more fitted to get insight into which factors act as a barriers or facilitators of adherence to treatment $(8,13)$. Considering the above-mentioned issues and also several years of work experience with $\beta$-TM patients, and dealing with challenges faced by this group of patients regarding their adherence to treatment, as well as dearth of qualitative research evidence concerning adherence to treatment in $\beta$-TM patients, the present study aimed to explore the perception of Iranian $\beta$-TM patients regarding the barriers and facilitators of adherence to their treatment.

\section{Material and Methods}

\subsection{Research design and participants}

This study was conducted by a qualitative research method using conventional content analysis for data analysis. Using purposive sampling, individuals with $\beta$-TM, requiring regular blood transfusion were eligible to participate in the study. Maximum variations in sampling in terms of different adherence level as well as various sociodemographic, economic and educational status were considered during the recruitment of the participants. Also, patients' family members and healthcare professionals who worked at thalassemia centers were interviewed using theoretical sampling. The number of interviews was guided by reaching data saturation. The study was conducted between August 2015 and May 2017 within Mazandaran Thalassemia Research Center, a specialized thalassemia treatment center in northern Iran, which is affiliated to the Mazandaran University of Medical Sciences, Sari, Iran.

\subsection{Data collection}

Semi-structured interviews were conducted by the first author (AEZ) who is a fourth-year doctoral student in nursing and was highly trained in interview processes and techniques, and was familiar with qualitative interview techniques. Participants were directly contacted and informed of the purpose of the study. All the interviews took place face-to-face at a date and time that was most convenient to participants, conducted in Persian and were audio taped. Interviews lasted between 30 and 95 minutes (mean: 57.11 minutes) and were guided by open-ended questions such as: "What do you usually do to control your disease?", "Can you tell me about how you manage your treatments?", "Have you experience any problems following up your prescribed treatments?" Participants were asked to share their experiences regarding main barriers to and facilitators of adherence to their prescribed treatment. The interviewer used probing questions to clarify a situation or to provide detail to an answer.

\subsection{Data analysis}

All the interviews were immediately transcribed verbatim. The transcripts were read by the authors several times to get insight into the participants' experiences. Thereafter, they were analyzed using conventional content analysis according to the technique described by Hsieh and Shannon (14). Data were coded, and related codes were finally grouped under certain categories by using the constant comparative analysis. The MAXQDA (Version 10) was used to organize, code, and manage the data.

\subsection{Ethical considerations}

Ethical approval for the study was obtained from an institutional ethics committee affiliated with Mashhad University of Medical Sciences (decree number: IR.MUMS.REC.1394.117). Participation in the present study was completely voluntary and informed written consent was obtained from all of them. All of the interviews were audio taped with the consent of the participants. 


\subsection{Rigor}

Lincoln and Guba's criteria for trustworthiness including credibility, transferability, dependability and confirmability, were used to ensure the rigor of the study (15). These criteria were established through a 21 -month engagement period in the research setting, providing thick descriptions illustrating the participants' lived experiences, peer debriefing, member checking, recording the decision trail throughout the data analysis process, recording the interviews and transcribing them immediately after each interview.

\section{Results}

In total, 26 interviews were conducted (12 men, 14 women, age range 13-50 years, mean age 33.9 years) with patients (10 men, 8 women), family members ( 3 mothers) and healthcare workers ( 3 nurses and 2 physicians) to reach data saturation. Among 18 patients, $6(33 \%), 8(44 \%)$ and $4(23 \%)$ patients had good, moderate and poor adherence to treatment, respectively. Also, 7 (339\%) of the patients were married. According to the participants' experiences, the major barriers of adherence to treatment were grouped into six interrelated categories; and main facilitators' themes were grouped into nine categories (Table 1).

Table 1. Barriers and facilitators of adherence to treatment in $\beta$-TM patients

\begin{tabular}{|l|l|}
\hline Barriers to adherence to treatment & Long distance challenges \\
\cline { 2 - 2 } & Delayed occurrence of complications \\
\cline { 2 - 2 } & Fatigue of healthcare providers \\
\cline { 2 - 2 } & Staff workload \\
\cline { 2 - 2 } & Insufficient follow-up of healthcare workers \\
\cline { 2 - 2 } & Busy everyday life \\
\hline & Concern of appearance changing \\
\cline { 2 - 2 } & Outcomes of treatment \\
\hline & Good awareness \\
\hline & Parental monitoring \\
\hline & Fear of complications \\
\hline & Social activity \\
\hline & Marriage as a facilitator \\
\cline { 2 - 2 } & Friendly interaction with the medical staff \\
\hline & Trusting the medical staff \\
\hline
\end{tabular}

\subsection{Barriers of adherence to treatment}

We identified several perceived barriers to patients' adherence to treatment which were classified into six main categories.

\subsubsection{Long distance challenges}

This category encapsulates participants' perceptions of concerns of long distance to treatment centers as a barrier to adherence to treatment. It was frequently discussed by both patients and healthcare workers, particularly for patients who lived in rural areas and need to travel long distances to access health facilities. A 28 -year-old male patient who lived in a village said: "Due to the long distance from our home to the thalassemia center, I am coming too late for blood transfusion. Well, I live in a village outside of the town" (Participant 3). Also, a physician who worked in the thalassemia center said: "I say to the patient's mother that your child has a problem in his liver, you must bring your child for testing. If you do not do it, there is a danger, he might lose his liver. My way is far away. I cannot bring him up to 3 weeks later; the mother said" (Participant 14).

3.1.2. Delayed occurrence of complications

Participants' interviews indicated that the relatively time-consuming nature of the occurrence of complications caused by non-adherence to treatment in $\beta$-TM patients, decrease their level of adherence to treatment. In this regard, a 31-year-old female patient with poor adherence to treatment stated that: "I did not follow-up my treatment and did not inject my desferal for about 4 months, because nothing happened for me in the months that I did not follow up this unpleasant treatment (Participant 4). A nurse who worked in the thalassemia center states that: "Usually the occurrence of complications caused by non-adherence to treatments in $\beta$-TM patients is timeconsuming (Participant 23)". 


\subsubsection{Fatigue of healthcare providers}

Participants' experiences in this study revealed that inappropriate following of healthcare providers' recommendations by the patients, can lead to the physician or nurses' fatigue, which consequently, leads to somewhat abandoning the patients' following up. This can impair patients' adherence to treatment. A 33 -year-old male patient with poor adherence to treatment said: "My mean ferritin level was more than 20000 during the last 9 months. Doctors and nurses told me very much that you must use your treatment regimen including desferal. But I ignored their recommendations and did not use the medications. So that they were tired and then did not do anything to me." (Participant 2). Also, a physician who worked in the thalassemia center stated: "Sometimes, well, some patients don't understand what you're saying. I see that the patient does not cooperate in any way we use. Well, I will be tired. I say, let it go" (Participant 22).

\subsubsection{Staff workload}

The participants' perspectives showed that a large number of patients together with the heavy workload of healthcare providers can lead to the fatigue of health professionals and impaired quality of healthcare services. Sometimes, lack of attention to the patients' concerns about their treatments are perceived by the patients during periodic visits by the physician. This can adversely affect the patient-physician relationship and consequently lead to non-adherence or irregular adherence to treatment. A 34-year-old female patient with good adherence to treatment states that: "When a patient asks a doctor something, it may not matter to them at that moment, because the number of patients are high and the doctor usually tries to ignore the details. Unless the patient, himself /herself, insists on the matter being dealt with." (Participant 1). Also, another 50-year-old female patient with moderate adherence said: "Well, the doctor does not have enough time to give a full explanation for me. She is busy. Often, she does not care what I'm saying" (Participant 17).

\subsubsection{Follow-up by healthcare providers}

According to the participants' perspective, if patients perceived that they do not receive appropriate follow-up after the prescribed treatment regimen by the healthcare providers, they felt that the received support by them is not sufficient to continue regularly their treatment, which could negatively affect their adherence. In this regard, a nurse who worked in the thalassemia center stated: "Some doctors do not show any reaction when the patient has not accomplished the recommended tests or prescribed medication. They do not follow up. The patient, thus, does not follow, when he sees, his doctor does not follow" (Participant 13).

3.1.6. Busy everyday life

According to the patients' experiences, a busy working day and complicated family life can lead to forgetting or postponing their treatments regimens. This issue was frequently discussed by married patients. A 33-year-old married male patient who had a child, stated: "Every day I must take our son to school and after that, I must get to the work on time. This is an everyday routine of my life which makes me so tired. Some days my tiredness results in that I do not care about my treatments or do it." (Participant 2).

\subsection{Facilitators of adherence to treatment}

Participants' interviews revealed several facilitators to patients' adherence to treatment which was classified into nine main categories.

\subsubsection{Concern of appearance changing}

Based on the patients' perspective, having a normal appearance and fear of face changing are facilitators of adherence to treatment. The changing appearance of thalassemia patients discloses their disease to the public; since, this disease has been known in the community by its typical facial deformity. This disclosure can lead to thalassemia related-stigma. Therefore, patients strive to follow their prescribed treatment regimen to avoid appearance changing. A 44-year-old mother with a child with $\beta$-TM who did not have any changes in her face, stated: "From the beginning of treatment, it had been told to me that if your child's hemoglobin levels fall below 10, his face will change. It was bearing this in my mind that I must bring him on time for receiving blood transfusion till his forehead and jaw bones do not protrude." (Participant 5). A 13-year-old male patient said: "I myself saw the color of my skin that really changed and became dark. So, I decided to decrease my ferritin level by injecting the desferal, regularly". (Participant 8). Another 37-year-old patients said: "I surely follow my treatment. I am giving hope to myself and trying not to change my face until when others see me, they too, do not understand that I am sick and have thalassemia". (Participant 10).

\subsubsection{Outcomes of treatment}

The experiences of thalassemic patients revealed that the occurrence of positive treatment's outcomes, in both patients and their peers, can motivate patients to follow their prescribed treatment regimens. In contrast, the negative outcomes, also, may strengthen the motivation of patients for reducing or preventing the serious adverse outcomes of the disease. In this regard, a 34-year-old female patient, with good adherence to treatment, stated: "I see that my 
friends who completely follow their treatment, do not have any complications. Well, it motivates me. On the other hand, when I see children who do not follow their treatment properly, they become problematic and may even die, it also makes me motivated to better follow up my treatment plan until those problems do not occur for me". (Participant).

\subsubsection{Good awareness}

Experience of participants demonstrated that good awareness of the disease and its treatment by the patients or their family members can play an important role in enhancing the adherence to treatment in $\beta$-TM patients. In this regard a 45-year-old female participant who had $\beta$-TM and worked in a thalassemia center as a nurse, stated: "When I was a child I do not think I ever forgot my blood transfusions or desferal. I think it is because my mother was a nurse and she knew why blood transfusion or desferal injection must be done in a timely manner" (Participant 15).

\subsubsection{Parental monitoring}

According to the experiences of participants, the parental monitoring and follow up facilitates adherence of patients to their prescribed regimen as well as motivates the patients to move forward with their treatment plan. A 25 -yearold female patient said: "My family follow-up has a great influence on me to keep up with my treatment and encourages me for desferal injection". (Participant 12). Also, a 29 -year-old male patient stated: "The parents' follow up is very important to my follow-up. My father still has prepared desferal for me. He always cares about my lab test and medicines. He even knows the number of past injected desferal that I have received since the start of my disease. It greatly motivates me to move forward." (Participant 24).

\subsubsection{Fear of complications}

Experiences of the participants revealed that their fear from the occurrence of treatment related complications such as blood transfusion induced iron overload as well as disease related complications, direct patients toward a comprehensive adherence to their treatment. A 21 -year-old male patient stated: "If I inject desferal irregularly, I may get diabetes, heart disease, and my liver may get hurt. For these, I inject the desferal regularly." (Participant 21). A 42-year-old mother of a child with $\beta$-TM said: "I regularly bring my son here to check his health status. I'm afraid of his liver enzyme raising and iron deposits around his heart." (Participant 7).

\subsubsection{Social activity}

Social activities, including getting to work, being with friends and acquaintances, being in the community and participating in social events encourage $\beta$-TM patients to better follow-up the prescribed treatment regimens. In this regard a 38-year-old male patient with moderate adherence to treatment stated: "Since I joined the community or I got to work, I did not want to get spoilt by others. Do you know, I do not want be always ill and be different from others. For all of these, I take my medications exactly as they prescribed." (Participant 26). Also, a 34-year-old female patient who worked in the thalassemia center said: "Since I have been working, my treatment's follow-up has been much better. I feel that my mood has been much better, and this seems to make me strictly follow the treatment's plan." (Participant 1).

\subsubsection{Marriage as a facilitator}

Marriage is one of the important motivators for patients to adhere to their treatment plan. Marriage and having a child sheds light on the future of thalassemia patients. After marriage, they commit themselves to regular follow-up of their prescribed treatments. The support of their spouse can ease the hardship of treatment. One of the participants who was a 33-year-old married male patient stated that: "Before I got married, I came to the thalassemia center very late and irregular. Believe me, my medical record is here. But, after marriage, I come here on a regular basis. Because I love my wife and children. This motivated me to follow-up my treatments." (Participant 2). Also, the head-nurse of the thalassemia center said: "One of the important things that motivate $\beta$-TM patients to adhere strictly to their treatment plan is marriage. I've seen a lot of these cases among our patients" (Participant 23).

\subsubsection{Friendly interaction with the medical staff}

Having a good relationship with medical staff was one the other important facilitators of adhering to the treatment plan in $\beta$-TM patients. In this regard a physician who worked in the thalassemia center said: "Those patients, with whom my relationship with them is better, are more likely to accept and follow my recommendations." (Participant 14). Also, a 42-year-old male patient said: "If doctors behave friendlier, I will follow-up their recommendations more, you know. Here, doctors and nurses' behaviors are good and this, encourages me to better follow the prescribed treatments." (Participant 20).

\subsubsection{Trusting the medical staff}

Trusting the medical staff has a great impact on patients' adherence to treatment. They believe that the medical staff usually do their best to improve patients' condition. In addition, most of the medical staff trust the patients. Damaging this mutual trust can disrupt adherence to treatment. In this regard, a physician who worked in the thalassemia center stated: "Patients are comfortable with me. They do not only look at me as a doctor. Most of them are friends with me. Maybe that is because I tell them everything, right or wrong. They trust me because many 
patients think that they are laboratory mice and doctors use them for research purposes. Since they trust me, they completely follow my recommendations." (Participant 22). Also, a 34-year-old female patient said: "Nurses gave us some pieces of advice and usually, we do what they say. Somehow, we trust nurses. We trust each other. We completely do anything that they recommend." (Participant 1).

\section{Discussion}

To the best of our knowledge this is the first in depth qualitative study exploring barriers and facilitators of adherence to treatment among $\beta$-TM patients in Iran. In this study, participants voiced a broad range of barriers and facilitators regarding their adherence to treatment. Difficulty of access to health care facilities, due to long distances, was one of the factors influencing adherence to treatment by $\beta$-TM patients. It has been previously shown that most $\beta$-TM patients are rural area residents and need to travel longer distances to access medical centers (16). Zamani et al. showed that the probability of long-term survival was significantly lower for $\beta$-TM patients who lived in rural areas, possibly due to their difficult access to healthcare facilities (17). Regular blood transfusion throughout life improves the prognosis of $\beta$-TM patients (18). Nonetheless, transfusion-related iron overload complications may be associated with higher mortality risk and shorter survival (19). The results of our study show that the fear of these complications is a potential facilitator to treatment adherence in $\beta$-TM patients. However, due to the delayed occurrence of complications, some patients may not follow their prescribed treatments regularly. It is believed that lack of immediate consequences of insufficient iron chelation therapy, unavoidably escalates the risk of developing iron overload complications in future $(10,20)$. In line with our findings, previous studies found that delay in the occurrence of complications can potentially lead to discontinued or irregular following of prescribed regimens by the patients $(18,19)$. Therefore, healthcare providers need to continually emphasize the importance of regular chelation therapy and assessment of body iron to patients for improving their survival chance $(18,19)$. Another two important barriers of adherence to treatment were staff's heavy workload and insufficient follow-up of healthcare providers. Consistent with our results, previously, the shortage of nursing staff and heavy workload has been reported in thalassemia centers $(21,22)$. Sapountzi-Krepia et al. showed that most of the $\beta$-TM patients' family members have been disappointed when asking for support and help from health professionals, because they are constantly busy (21). It has been previously revealed that healthcare providers' heavy workload is a major barrier to patients' education, counseling and supervision (23). Despite their overwhelming workload, most healthcare providers try to offer reasonable recommendations to patients for regular treatment follow up. As a consequence, discontinuation or irregular following of the treatment plan by patients can lead to healthcare providers' fatigue and discourage them from giving further recommendations to patients $(24,25)$. Concern of appearance changing was one of the facilitators of adherence to treatment by $\beta$-TM patients. In $\beta$-TM patients, typical facial deformities play an important role in their social stigmatization which can have potential negative consequences (26). Wahab et al. and Pouraboli et al. also showed similar areas of concern in $\beta$-TM patients $(27,28)$. It has been demonstrated that persons with facial changes experience staring and stigmatization as negative reactions (29). Treatments' outcome was another facilitator of adherence to treatment in $\beta$-TM patients. It has been revealed that the favorable outcomes of treatment increase the likelihood of adherence (30). In our study, good awareness of the disease and treatments has been revealed as a facilitator of treatment adherence in $\beta$-TM patients. Lee et al. showed a significant positive correlation between levels of disease knowledge in $\beta$-TM patients and their mothers with treatment adherence (31). Al-Kloub et al. showed that patients' disease knowledge is not associated with adherence status of adolescents with $\beta$-TM (32). Adequate knowledge about disease and treatment is essential but not sufficient for appropriate adherence to prescribed treatment regimen, and does not necessarily lead to improved patients' adherence. Several other potential factors may have also contributed to poor adherence in patients with chronic diseases $(19,32)$.

The results of this study also revealed that family support is a facilitator of adherence to treatment in $\beta$-TM patients which is consistent with the findings of previous studies $(32,33)$. Several studies showed that the lack of family involvement in the following and monitoring of activities related to the treatment regimens can negatively affect patients' adherence to their therapeutic plan $(34,35)$. It has previously been shown that a low level of social activity is a barrier of adherence to treatment (36). This is important because thalassemia related-stigma most probably is associated with emotional, mental and social disadvantages in $\beta$-TM patients. Social stigma can lead to patients' rejection, isolation and unemployment (28). In this regard, previous studies showed that patients' adherence to their prescribed medical regimen may be influenced by their employment status (37). Employment is associated with better psychosocial well-being and social support. Unemployment is usually associated with impaired psychosocial well-being and mental health which consequently may lead poor adherence to treatment $(37,38)$. The results of our study showed that transition from being single to being married was associated with increased adherence to treatment in $\beta$-TM patients. Married patients are more likely to strictly adhere to their prescribed treatment than 
unmarried patients. A possible explanation for this is that being supported by relatives, particularly his/her spouse can be a motivator for $\beta$-TM patients to continuing lifelong treatment plans. Friendly interaction with the medical staff and trusting them are other perceived facilitators by our participants. This finding is supported by previous studies that highlight the importance of good trust in healthcare professionals and also good communication between them in improving adherence to treatment in chronic diseases $(32,39,40)$. There are some limitations in the present study that need to be addressed. In this study, all participants were enrolled from one thalassemia center in northern Iran. Therefore, the results may not be generalized to all $\beta$-TM population. Additionally, the responses of participants regarding adherence to their treatment might have been influenced by social acceptability bias, although an attempt was made to minimize this bias during interviews.

\section{Conclusions}

The results of this study provide a broad range of context-specific facilitators and barriers to treatment adherence perceived by Iranian $\beta$-TM patients. These factors can improve our understanding of the complex and multifactorial nature of adherence to treatments in $\beta$-TM patients. Health care providers can use these finding in designing and implementing appropriate interventions to promote $\beta$-TM patients' adherence to treatment. Therefore, further welldesigned clinical trials in this regard are warranted.

\section{Acknowledgments:}

This study is a part of the first author's doctoral dissertation. The financial support of Deputy of Research and Technology of Mashhad University of Medical Sciences (code: 931582) is gratefully acknowledged. Also, the authors wish to thank all the study participants for their tremendous cooperation and support.

\section{Conflict of Interest:}

All authors declare no conflict of interest; no conflict of interest exists for any of the authors associated with the manuscript. The funding organization had no role in the design and conduct of the study, or in the collection, analysis, and interpretation of the data.

\section{Authors' contributions:}

Study conception and design: AEZ, HKM, and AH. Acquisition of data: AEZ. Interpretation of data: AEZ, HKM, and AH. Drafting of the manuscript: AEZ, HKM, and AH. Critical revision of the manuscript for important intellectual content: AEZ, HKM, and AH.

\section{References:}

1) Mednick L, Yu S, Trachtenberg F, Xu Y, Kleinert DA, Giardina PJ, et al. Symptoms of depression and anxiety in patients with thalassemia: prevalence and correlates in the thalassemia longitudinal cohort. Am J Hematol. 2010; 85(10): 802-5. doi: 10.1002/ajh.21826. PMID: 20806230.

2) Darvishi Khezri H, Emami Zeydi A, Sharifi H, Jalali H. Is Vitamin C Supplementation in Patients with beta-Thalassemia Major Beneficial or Detrimental? Hemoglobin. 2016; 40(4): 293-4. doi: 10.1080/03630269.2016.1190373. PMID: 27492769.

3) Dehshal MH. Addressing adherence to treatment: a longstanding concern. The patients' perspective. Thalassemia Reports. 2014; 4(3). doi: 10.1080/03630269.2016.1190373. PMID: 27492769.

4) Vermeire E, Hearnshaw H, Van Royen P, Denekens J. Patient adherence to treatment: three decades of research. A comprehensive review. J Clin Pharm Ther. 2001; 26(5): 331-42. doi: 10.1046/j.13652710.2001.00363.x. PMID: 11679023.

5) Pedram M, Zandian K, Keikhaie B, Akramipour R, Hashemi A, Ghahfarokhi FK, et al. A report on chelating therapy and patient compliance by determination of serum ferritin levels in 243 thalassemia major patients. Iranian J Pediatr Soc. 2010; 2(2): 65-9.

6) Ragab LA, Hamdy MM, Shaheen IA, Yassin RN. Blood transfusion among thalassemia patients: A single Egyptian center experience. Asian J Transfus Sci. 2013; 7(1): 33-6. doi: 10.4103/0973-6247.106728. PMID: 23559762.

7) Coifman KG, Ross GS, Kleinert D, Giardina P. Negative affect differentiation and adherence during treatment for thalassemia. Int J Behav Med. 2014; 21(1): 160-8. PMID: 23086716. doi: 10.1007/s12529012-9277-7.

8) Pages-Puigdemont N, Mangues MA, Masip M, Gabriele G, Fernandez-Maldonado L, Blancafort S, et al. Patients' Perspective of Medication Adherence in Chronic Conditions: A Qualitative Study. Adv Ther. 2016; 33(10): 1740-54. PMID: 27503082. doi: 10.1007/s12325-016-0394-6. 
9) Lehane E, McCarthy G. Intentional and unintentional medication non-adherence: a comprehensive framework for clinical research and practice? A discussion paper. Int J Nurs Stud. 2007; 44(8): 1468-77. doi: 10.1016/j.ijnurstu.2006.07.010. PMID: 16973166.

10) Ward A, Caro JJ, Green TC, Huybrechts K, Arana A, Wait S, et al. An international survey of patients with thalassemia major and their views about sustaining life-long desferrioxamine use. BMC Clin Pharmacol. 2002; 2: 3. doi: 10.1186/1472-6904-2-3. PMID: 12015817.

11) Morris LS, Schulz RM. Medication compliance: the patient's perspective. Clin Ther. 1993; $15(3): 593-606$. PMID: 8364951.

12) Horne R, Weinman J. Patients' beliefs about prescribed medicines and their role in adherence to treatment in chronic physical illness. J Psychosom Res. 1999; 47(6): 555-67. doi: 10.1016/S0022-3999(99)00057-4. PMID: 10661603.

13) Al-Busaidi ZQ. Qualitative research and its uses in health care. Sultan Qaboos Univ Med J. 2008; 8(1): 11 9. PMID: 21654952.

14) Hsieh HF, Shannon SE. Three approaches to qualitative content analysis. Qual Health Res. 2005; 15(9): 1277-88. doi: 10.1177/1049732305276687. PMID: 16204405.

15) Lincoln YS, Guba EG. But is it rigorous? Trustworthiness and authenticity in naturalistic evaluation. New Directions for Program Evaluation. 1986; 1986(30): 73-84. doi: 10.1002/ev.1427.

16) Shaligram D, Girimaji SC, Chaturvedi SK. Psychological problems and quality of life in children with thalassemia. Indian J Pediatr. 2007; 74(8): 727-30. doi: 10.1007/s12098-007-0127-6. PMID: 17785893.

17) Zamani R, Khazaei S, Rezaeian S. Survival analysis and its associated factors of Beta thalassemia major in hamadan province. Iran J Med Sci. 2015; 40(3): 233-9. PMID: 25999623.

18) Ganzella M, Zago MM. The experience of thalassemic adults with their treatment. Rev Lat Am Enfermagem. 2011; 19(4): 968-76. doi: 10.1590/S0104-11692011000400016. PMID: 21876950.

19) Borgna-Pignatti C, Gamberini MR. Complications of thalassemia major and their treatment. Expert Rev Hematol. 2011; 4(3): 353-66. doi: 10.1586/ehm.11.29. PMID: 21668399.

20) Olivieri NF. Adherence to deferoxamine therapy: heeding Hippocrates and Osler. Am J Hematol. 2004; 76(4): 415-6. doi: 10.1002/ajh.20102. PMID: 15282681.

21) Sapountzi-Krepia D, Roupa Z, Gourni M, Mastorakou F, Vojiatzi E, Kouyioumtzi A, et al. A qualitative study on the experiences of mothers caring for their children with thalassemia in Athens, Greece. J Pediatr Nurs. 2006; 21(2): 142-52. doi: 10.1016/j.pedn.2005.06.017. PMID: 16545674.

22) Miri-Moghaddam E, Naderi M, Izadi S, Mashhadi M. Causes of new cases of major thalassemia in sistan and balouchistan province in South-East of iran. Iran J Public Health. 2012; 41(11): 67-71. PMID: 23304678 .

23) Karimi Moonaghi H, Emami Zeydi A, Mirhaghi A. Patient education among nurses: bringing evidence into clinical applicability in Iran. Invest Educ Enferm. 2016; 34(1): 137-51. doi: 10.17533/udea.iee.v34n1a16. PMID: 28569983.

24) van Ryn M, Burke J. The effect of patient race and socio-economic status on physicians' perceptions of patients. Soc Sci Med. 2000; 50(6): 813-28. doi: 10.1016/S0277-9536(99)00338-X. PMID: 10695979.

25) M'Imunya JM, Kredo T, Volmink J. Patient education and counselling for promoting adherence to treatment for tuberculosis. Cochrane Database Syst Rev. 2012(5):Cd006591. doi: 10.1002/14651858.CD006591.pub2. PMID: 22592714.

26) Loizou C, Phellas C, Beck S, Karekla M, Talias M, Christou S, et al. How Life Would Be Without Thalassaemia: Patients' Perceptions. The Cyprus Review. 2016; 28(1): 33.

27) Wahab IA, Naznin M, Nora MZ, Suzanah AR, Zulaiho M, Faszrul AR, et al. Thalassaemia: a study on the perception of patients and family members. Med J Malaysia. 2011; 66(4): 326-34. PMID: 22299552.

28) Pouraboli B, Abedi HA, Abbaszadeh A, Kazemi M. Living in a misty marsh: A qualitative study on the experiences of self-care suffering of patients with thalassemia. Iran J Nurs Midwifery Res. 2014; $19(7$ Suppl 1):S77-82. PMID: 25949257, PMCID: PMC4402994.

29) Rumsey N, Harcourt D. Visible difference amongst children and adolescents: issues and interventions. Dev Neurorehabil. 2007; 10(2): 113-23. doi: 10.1080/13638490701217396. PMID: 17687984.

30) DiMatteo MR, Giordani PJ, Lepper HS, Croghan TW. Patient adherence and medical treatment outcomes: a meta-analysis. Med Care. 2002; 40(9): 794-811. doi: 10.1097/01.MLR.0000024612.61915.2D. PMID: 12218770.

31) Lee YL, Lin DT, Tsai SF. Disease knowledge and treatment adherence among patients with thalassemia major and their mothers in Taiwan. J Clin Nurs. 2009; 18(4): 529-38. doi: 10.1111/j.13652702.2007.02150.x. PMID: 19192002. 
32) Al-Kloub MI, A Bed MA, Al Khawaldeh OA, Al Tawarah YM, Froelicher ES. Predictors of non-adherence to follow-up visits and deferasirox chelation therapy among Jordanian adolescents with Thalassemia major. Pediatr Hematol Oncol. 2014; 31(7): 624-37. doi: 10.3109/08880018.2014.939792. PMID: 25116329.

33) Ellis DA, Podolski CL, Frey M, Naar-King S, Wang B, Moltz K. The role of parental monitoring in adolescent health outcomes: impact on regimen adherence in youth with type 1 diabetes. J Pediatr Psychol. 2007; 32(8): 907-17. doi: 10.1093/jpepsy/jsm009. PMID: 17426045.

34) Wiebe DJ, Berg CA, Korbel C, Palmer DL, Beveridge RM, Upchurch R, et al. Children's appraisals of maternal involvement in coping with diabetes: enhancing our understanding of adherence, metabolic control, and quality of life across adolescence. J Pediatr Psychol. 2005; 30(2): 167-78. doi: 10.1093/jpepsy/jsi004. PMID: 15681311.

35) Michaud PA, Suris JC, Viner R. The adolescent with a chronic condition. Part II: healthcare provision. Arch Dis Child. 2004; 89(10): 943-9. doi: 10.1136/adc.2003.045377. PMID: 15383439.

36) Jack K, McLean SM, Moffett JK, Gardiner E. Barriers to treatment adherence in physiotherapy outpatient clinics: a systematic review. Man Ther. 2010; 15(3): 220-8. PMID: 20163979. doi: 10.1016/j.math.2009.12.004.

37) Nachega JB, Uthman OA, Peltzer K, Richardson LA, Mills EJ, Amekudzi K, et al. Association between antiretroviral therapy adherence and employment status: systematic review and meta-analysis. Bull World Health Organ. 2015; 93(1): 29-41. doi: 10.2471/BLT.14.138149. PMID: 25558105.

38) Herbig B, Dragano N, Angerer P. Health in the long-term unemployed. Dtsch Arztebl Int. 2013; $110(23-$ 24): 413-9. doi: 10.3238/arztebl.2013.0413. PMID: 23837086.

39) Simba DO, Kakoko D. Primacy of effective communication and its influence on adherence to artemetherlumefantrine treatment for children under five years of age: a qualitative study. BMC Health Serv Res. 2012; 12: 146. doi: 10.1186/1472-6963-12-146. PMID: 22682180.

40) Birkhauer J, Gaab J, Kossowsky J, Hasler S, Krummenacher P, Werner C, et al. Trust in the health care professional and health outcome: A meta-analysis. PLoS One. 2017; 12(2): e0170988. doi: 10.1371/journal.pone.0170988. PMID: 28170443. 